\title{
A SPECTROSCOPIC SURVEY FOR BINARY STARS IN THE GLOBULAR CLUSTER NGC 5053
}

\author{
LIN YAN ${ }^{1}$ AND J. G. COHEN \\ Department of Astronomy, MS 105-24, California Institute of Technology, Pasadena, California 91125 \\ Electronic mail: lyan@eso.org, jlc@astro.caltech.edu \\ Received 1996 February 6; revised 1996 June 16
}

\begin{abstract}
We carried out a radial velocity survey for spectroscopic binaries in the low density globular cluster NGC 5053. Our sample contains a total of 77 cluster member giant and subgiant stars with visual magnitudes of 14.5-18.6. Of these 77 stars, 66 stars have on average of 3-4 measurements with a total of 236 velocities. A typical velocity error per measurement is $\sim 3 \mathrm{~km} \mathrm{~s}^{-1}$. The stars in our sample are spatially distributed from the cluster center out to 10 arcmin in radius (4.5 core radii). Among these 66 stars with multiple velocity measurements, we discovered 6 spectroscopic binary candidates. Of these six candidates, one was discovered as a binary previously by Pryor et al. (1991) and candidate ST is a binary with a very short period of three to five days. We obtained three possible orbital solutions for binary candidate ST by fitting its radial velocity data. These orbital solutions are consistent with star ST being a cluster member, although its spectrum has much stronger $\mathrm{Mg}$ I triplet absorption lines than that of a typical low-metallicity giant star. Using a Monte Carlo simulation method, we estimated the fraction of binary systems which may have been missed from our detection due to unfavorable orbital configurations. With our survey, the binary discovery efficiency is $29 \%$ for systems with $3 \mathrm{~d} \leqslant P \leqslant 10 \mathrm{yr}, 0.125 \leqslant q \leqslant 1.75$ and eccentric orbits $(0 \leqslant e \leqslant 1)$. This yields a binary frequency of $29 \%$. We also applied Kolmogorov-Smirnov (K-S) tests to the cumulative distributions of maximum velocity variations from the actual measurements and the synthetic velocity data. The results from these tests are consistent with $21 \%-29 \%$ binary population with $3 \mathrm{~d} \leqslant P \leqslant 10 \mathrm{yr}$, $0.125 \leqslant q \leqslant 1.75$ in NGC 5053. The hypothesis of a binäry frequency in NGC 5053 higher than $50 \%$ is rejected with a confidence level higher than $85 \%$. The binary frequency in NGC 5053 derived from our survey is somewhat higher than estimates for other clusters by various surveys. This is perhaps related to the fact that NGC 5053 is relatively dynamically young compared to other clusters. We also argue that the binary population in globular clusters is not significantly deficient compared to binaries in other stellar environments such as open clusters, or to field and low metallicity halo stars. () 1996 American Astronomical Society.
\end{abstract}

\section{INTRODUCTION}

Recently, it has been realized that a primordial binary frequency as small as $3 \%$ can fundamentally change the dynamical evolution of an entire globular cluster (Heggie \& Aarseth 1992). Mass segregation caused by two-body relaxation in a cluster essentially transfers "heat" (stellar kinetic energy) from the cluster core to the "cooling" edges, while simultaneously pushing the cluster towards higher central concentration (gravothermal collapse). The process of gravothermal collapse can be greatly modified by a binary star population (Gao et al. 1991; Heggie \& Aarseth 1992; McMillan \& Hut 1994; Vesperini \& Chernoff 1994). Gravitational binding energy in binary stars can be extracted and converted into kinetic energy during encounters with other stars. The extracted energy can supply a central heat source to stave off or reverse gravothermal collapse.

There is considerable observational evidence that binary stars do exist in globular clusters (see the detailed review by Hut et al. 1992). Discoveries of low-mass x-ray binaries

${ }^{1}$ Present address: European Southern Observatory: Karl-Schwarzschild-Str. 2, D-85748 Garching b. München, Germany.
(LMXB) in globular clusters (Grindlay et al. 1984) first hinted that a primordial binary population could exist in globular clusters, although the formation of LMXBs does not necessarily require the pre-existing binary populations. Indisputable evidence came from the discovery of long-period binary millisecond pulsars in low density globular clusters during the late 80 's and the early 90's. Among a total of $\sim 40$ millisecond pulsars in globular clusters, $\sim 12$ of them are actually in binary systems. Furthermore, two of these binary millisecond pulsars-discovered in the low density clusters M4 and M53-have periods as long as 200-300 days (McKenna \& Lyne 1988; Kulkarni et al. 1991). Binaries with such long periods cannot be formed through single star-star tidal capture. In fact, PSR $1640-26$ in M4 is a triple system. These two long-period millisecond pulsars either have to be formed by star-binary and binary-binary encounters, or their progenitors are primordial binary stars.

Recent direct searches for spectroscopic and short-period eclipsing binaries have discovered many binary systems and binary candidates in globular clusters (Pryor et al. 1991; Côte et al. 1994; Mateo et al. 1990; Yan \& Mateo 1994; Yan \& Reid 1995). One of the drivers behind these efforts is to understand many apparently different phenomena in globular 
clusters which may be intimately related to binary star population. For example, such phenomena include blue stragglers, color/stellar gradient in the cores of post-core-collapsed clusters (Djorgovski \& King 1986), x-ray sources and numerous "recycled" radio pulsars (Phinney 1992, 1996). The discovery of short-period eclipsing binaries among both blue straggler stars and main-sequence stars (Mateo et al. 1990; Kaluzny \& Krzeminski 1993; Yan \& Mateo 1994; Yan \& Reid 1995) suggests that the short-period binaries may be the progenitors of blue stragglers. Mass transfer/merger among stars in binary systems and stellar encounters involving binary stars are important processes for the formation of blue stragglers. Also, numerous X-ray sources and radio pulsars in globular clusters can be easily explained in terms of binary systems containing degenerate stars.

While mounting evidence, direct or indirect, has suggested the existence of both short- and long-period binary stars in globular clusters, the binary frequency and binary period distributions are very poorly determined. This has been the major source of uncertainty in the theoretical modeling the role of binaries in the evolution of globular clusters. For binaries with periods in the range of 10 days to 10 years, the direct way to determine the binary frequency is to systematically measure radial velocity variations in individual stars of a globular cluster. Technically only recently with the advent of high-resolution multiobject spectrographs on $4 \mathrm{~m}$ class telescopes did it become possible for the first time to sample a large number of stars and to obtain multiepoch observations with a reasonable amount of telescope time. With telescopes smaller than the Keck $10 \mathrm{~m}$ telescope, we are limited to bright red giants and subgiants even in the closest globular cluster. These bright low metallicity giant stars have smaller masses and larger radii compared to the field G-type stars. Consequently, a binary system containing such a bright giant star is difficult to detect since the system tends to have a long period and small radial velocity variations.

All of the existing radial velocity surveys of binary stars in globular clusters generally were done among the bright giant stars (Gunn \& Griffin 1979; Pryor et al. 1988; Côte et al. 1994). The first radial velocity survey with high precision was done by Gunn \& Griffin (1979) in the globular cluster M3. They obtained a total of 85 velocity measurements for 33 giant stars with $V$ magnitudes 12-14. They failed to find any binary candidates among the nonpulsating stars and concluded that binaries with separations between 0.3 and $10 \mathrm{AU}$ (periods between 0.1 and $30 \mathrm{yr}$ ) were much rarer in globular clusters than in the field Population I stars. In 1988, combining Gunn \& Griffin's data in M3 with new MMT observations, Pryor et al. produced a larger dataset containing 111 giant stars, each with 3 multiple measurements. They found one binary candidate, which later was confirmed as a system with a period of $7.3 \mathrm{yr}$. Recently, Côte et al. (1994) published a similar survey in the globular cluster NGC 3201. A total of 786 velocities were obtained for 276 giant stars with $V$ magnitudes of 11.0-16.5 over a total timespan of $6 \mathrm{yr}$. They found 2 good binary candidates, plus 13 possible candidates. The derived binary frequency from both surveys is roughly $5 \%-18 \%$ for systems with 0.1 $\mathrm{yr} \leqslant P \leqslant 10 \mathrm{yr}$ and $0.1 \leqslant q \leqslant 1.0$, depending on orbital eccentricities.

With the advent of the Norris Multi-fiber Spectrograph on the 200 in. telescope at the Palomar observatory, we carried out a radial velocity survey for spectroscopic binary stars in the globular cluster NGC 5053. Our survey was designed to reach much fainter stars than previous surveys. It is thus sensitive to binary systems with much shorter periods, and much larger radial velocity variations. The object sample in our survey consists of 77 cluster member giant and subgiant stars in the globular cluster NGC 5053 with visual magnitudes in the range of 14.2-18.6. We have obtained 6 epochs of observations of these 77 stars in a total timespan of $3 \mathrm{yr}$. Compared to the previous radial velocity survey made in the same cluster by Pryor et al. (Pryor et al. 1991), our survey samples more than twice as many stars and goes almost three magnitude fainter. Our survey is sensitive to binary systems with periods as short as 3 days. For potential binaries in our sample, a maximum radial velocity variation as large as 116 $\mathrm{km} \mathrm{s}^{-1}$ is possible for an edge-on circular orbit with $M_{1}$ and $M_{2}$ of 0.8 and $0.5 \mathscr{C}_{\odot}$, respectively. Our velocity measurement error varies from star to star, but typically is about 3 $\mathrm{km} \mathrm{s}^{-1}$, which is somewhat worse than in previous surveys (We discuss in detail the velocity measurement errors and related problems in Sec. 2.2). However, the smaller radii of the stars in our sample compensates for this disadvantage. In addition, our sample stars are less luminous and do not have radial velocity variations on a scale of $8 \mathrm{~km} \mathrm{~s}^{-1}$ caused by intrinsic atmospheric motions found among bright giant stars by previous surveys (Gunn \& Griffin 1979; Lupton et al. 1987; Pryor et al. 1988).

The globular cluster NGC 5053 is an ultralow density cluster with a half-mass relaxation time scale of $\sim 8 \mathrm{Gyr}$ (Djorgovski 1992), comparable to the Hubble time scale. This suggests that this cluster is barely dynamically relaxed, and the spatial and period distribution of its primordial binary population has not been significantly altered by twobody relaxation processes. Moreover, the long relaxation timescale indicates that in this cluster the binary destruction by star-binary and binary-binary encounters is not important. However, one disadvantage imposed on our survey is that NGC 5053 is a very metal poor cluster with $[\mathrm{Fe} / \mathrm{H}]$ of -2.2 , and thus the spectra from its stars have weaker metal absorption lines than metal-rich objects.

\section{THE SURVEY}

\subsection{Observations}

All of the observations were made with the Norris Multifiber Spectrograph on the Hale $5.0 \mathrm{~m}$ telescope at the Palomar observatory. The Norris Spectrograph is a fiber-fed multiobject spectrograph which is mounted at the Cassagrin focal plan of the telescope (Hamilton et al. 1993). It has a total of 176 fibers, and each fiber has a 1.6 arcsec diameter aperture. The small diameter of the fiber aperture implies that the effective throughput of the spectrograph is very sensitive to seeing conditions, especially for stellar objects, as in our case. The fibers are located in two opposing banks of equal number. The minimum separation between two fibers 


\begin{tabular}{|c|c|c|c|c|c|}
\hline $\begin{array}{l}\text { Date } \\
\text { (UT) }\end{array}$ & Object & $\begin{array}{l}\text { Exp. Time } \\
\text { (sec) }\end{array}$ & $\begin{array}{l}\Delta \lambda \\
(\AA)\end{array}$ & $\begin{array}{l}\text { Resoluti } \\
\qquad(\AA)\end{array}$ & $\begin{array}{l}\text { Weather } \\
\text { conditions }\end{array}$ \\
\hline $7 / 4 / 1992$ & NGC 5053 & $3 \times 3000$ & $4710-5310$ & 2.5 & clear, $1.3^{\prime \prime}$ seeing \\
\hline $7 / 4 / 1992$ & M 13 & $1 \times 900$ & $4710-5310$ & 2.5 & clear, $1.3^{\prime \prime}$ seeing \\
\hline $8 / 4 / 1992$ & NGC $5053^{2}$ & $3 \times 3000$ & $4710-5310$ & 2.5 & cloudy, $1.3^{\prime \prime}$ seeing \\
\hline $8 / 4 / 1992$ & M 13 & $1 \times 900$ & $4710-5310$ & 2.5 & cloudy, $1.3^{\prime \prime}$ seeing \\
\hline $25 / 5 / 1992$ & NGC 5053 & $2 \times 3000$ & $4710-5310$ & 2.5 & cloudy, $1.5^{\prime \prime}$ seeing \\
\hline $26 / 5 / 1992$ & NGC 5053 & $3 \times 3000$ & $4710-5310$ & 2.5 & clear, $1.5^{\prime \prime}$ seeing \\
\hline $26 / 5 / 1992$ & M 13 & $1 \times 900$ & $4710-5310$ & 2.5 & clear, $1.5^{\prime \prime}$ seeing \\
\hline $16 / 4 / 1993$ & NGC 5053 & $1 \times 3000$ & $4710-5310$ & 2.5 & cloudy, $1.5^{\prime \prime}$ seeing \\
\hline $16 / 4 / 1993$ & M 13 & $1 \times 900$ & $4710-5310$ & 2.5 & cloudy, $1.5^{\prime \prime}$ seeing \\
\hline $21 / 5 / 1993$ & NGC 5053 & $1 \times 2515$ & $4710-5310$ & 2.5 & cloudy, $1.5^{\prime \prime}$ seeing \\
\hline $21 / 5 / 1993$ & M 13 & $1 \times 900$ & $4710-5310$ & 2.5 & cloudy, $1.5^{\prime \prime}$ seeing \\
\hline $11 / 4 / 1994$ & NGC 5053 & $3 \times 3000$ & $4700-5800$ & 3.0 & clear, $1.2^{\prime \prime}$ seeing \\
\hline $11 / 4 / 1994$ & M 13 & $2 \times 700$ & $4700-5800$ & 3.0 & clear, $1.2^{\prime \prime}$ seeing \\
\hline $12 / 4 / 1994$ & NGC 5053 & $3 \times 3000$ & $4700-5800$ & 2.5 & cloudy, $1.5^{\prime \prime}$ seeing \\
\hline $12 / 4 / 1994$ & M 13 & $3 \times 900$ & $4700-5800$ & 3.0 & cloudy, $1.5^{\prime \prime}$ seeing \\
\hline $12 / 4 / 1994$ & NGC $5053^{2}$ & $4 \times 3000$ & $4700-5800$ & 3.0 & cloudy, $1.5^{\prime \prime}$ seeing \\
\hline $8 / 5 / 1994$ & NGC 5053 & $3 \times 3000$ & $4700-5800$ & 2.5 & cloudy, $1.3^{\prime \prime}$ seeing \\
\hline $8 / 5 / 1994$ & M 13 & $1 \times 600$ & $4700-5800$ & 2.5 & cloudy, $1.3^{\prime \prime}$ seeing \\
\hline $10 / 5 / 1994$ & NGC 5053 & $2 \times 3000$ & $4700-5800$ & 2.5 & clear, $1.2^{\prime \prime}$ seeing \\
\hline $10 / 5 / 1994$ & M 13 & $3 \times 600$ & $4700-5800$ & 2.5 & clear, $1.5^{\prime \prime}$ seeing \\
\hline $10 / 5 / 1994$ & NGC $5053^{2}$ & $2 \times 3000$ & $4700-5800$ & 3.0 & cloudy, $1.5^{\prime \prime}$ seeing \\
\hline $10 / 5 / 1994$ & M 13 & $1 \times 900$ & $4700-5800$ & 2.5 & clear, $1.3^{\prime \prime}$ seeing \\
\hline
\end{tabular}

Notes: NGC $5053^{2}$ is the second field we observed in the same cluster. In M 13 several bright giant stars were observed to serve as the radial velocity standards. The radial velocities of these stars have been accurately measured by Lupton, Gunn and Griffin (1987) The third column in the table gives the number of exposure times the time of a single exposure, i.e. the total exposure time. The spectral resolution in April 1994 is larger than other runs due to problems with the collimator focus in the Norris spectrograph during that observing run. The CCD spectral scale is $0.65 \AA$ pixel $^{-1}$ for all of the observations.

is $\sim 16$ arcsec, which is the major limitation for sampling more stars in globular clusters. The spectrograph covers a field of view of $20 \times 20$ square arcminutes.

We obtained a total of 6 epochs of observations of a sample of 77 cluster member stars with a total timespan of 3 yr. The summary of all observations is tabulated in Table 1. The first four epochs of observations in 1992 and 1993 were taken with a $1024 \times 1024$ pixels CCD and the field of view of $10 \times 10$ square arcminutes. The last two epochs of observations in 1994 were taken with a more sensitive and larger CCD with $2048 \times 2048$ pixels. These observations therefore had wider wavelength coverage and sampled more stars. Thus some of the stars in our sample have more velocity measurements than others. Also notice that the total exposure time for the primary field in NGC 5053 varies from one epoch to another due to changes in the amount of available observing time and the observing conditions. For some epochs, we could not obtain any observations for the second field in the same cluster. This is one of the causes for the variations of velocity measurement errors for the same star at different epochs.

During all observations, we used a 1200 groove/mm grating centered around $5000 \AA$. The resulting spectral scale is $\sim 0.65 \AA /$ pixel, corresponding to the velocity scale of $\sim 39$ $\mathrm{km} \mathrm{s}^{-1}$ pixel $^{-1}$. The spectral resolution of the observations is around 2.5-3 $\AA$. With a cross-correlation technique, we should be able to measure any velocity shift larger than one tenth of a pixel, i.e., $4 \mathrm{~km} \mathrm{~s}^{-1}$. The poor resolution in the 1994 April observing run was due to bad collimator focus caused by a mechanical problem in the spectrograph.

To make accurate velocity measurements, in all our observations we took comparison Thorium-Argon spectra both before and after each object exposure to calibrate out any spectrograph flexure. Dome flats were taken immediately after each setup to flatfield the object spectra. We also obtained some exposures of the twilight sky to check the spectrograph velocity zero-point shifts between different nights and also between different observing runs. Depending on the observing conditions and the amount of time we had during each run, we observed at least the primary field and sometimes the secondary field in NGC 5053. Since it is relative velocity variations which we need to measure accurately in the search for spectroscopic binaries, we chose about ten giant stars brighter than $13 \mathrm{mag}$ in the globular cluster M13 to serve as the velocity standards. These giant stars were chosen from the sample in the radial velocity survey by Lupton et al. (1987). The typical velocity error in their survey is $\sim 1$ $\mathrm{km} \mathrm{s}^{-1}$. One advantage of using these stars is that we were able to obtain all their spectra with a single exposure.

\subsection{Data Reduction}

Our data were reduced using IRAF. ${ }^{2}$ After the images were trimmed and corrected for bias, all of the object spectra, including sky exposures, were identified, traced and extracted using APALL task in the IRAF package SPECRED. An optimal extraction algorithm was used to produce onedimensional spectra for all stars. The dome-flat spectra from all fibers were extracted and averaged. A low-order polynomial was used to fit the averaged dome-flat spectrum. The flat-field spectra were obtained by normalizing all dome-flat spectra with the single fitted spectrum. The CCD sensitivity variations at small scales in all object spectra were taken out by dividing them with the corresponding flat-field spectra.

Figures 1(a) and 1(b) show spectra of the same star with a signal-to-noise ratio of $\sim 15$, taken in 1992 and 1994, respectively. Signal-to-noise ratios of our spectra vary within a large range, but the typical value of a continuum is around 10. The spectra taken in 1994 have the wavelength coverage of 4700-5800 $\AA$, and consequently include very strong sky emission lines such as [O I] $5577 \AA$, Hg I $5461 \AA$, and $\mathrm{Hg}$ doublet $5791 \AA$, and the Na D doublet $5893 \AA$. To obtain good sky subtraction, we corrected the variations of throughput from fiber to fiber. The correction for each fiber was estimated by dividing the integrated flux in the sky emission line $\mathrm{Hg}$ I $5461 \AA$ with the average flux of the same line from all fibers. The most useful absorption hines for doing radial velocity cross correlation, $\mathrm{H}_{\beta} 4861 \AA$ and the $\mathrm{Mg}$ I triplet at 5167, 5173, and $5184 \AA$, are outside the strong sky emission line region. Residuals of sky emission lines in the skysubtracted spectra do not have a big effect on the velocity measurement. In the cases of large residuals from the sky subtraction, we simply mask off the regions with sky lines. Throughput correction was not necessary for spectra taken in 1992 since there are not many strong sky emission lines in the range of $4710-5300 \AA$. Figure 1 (c) shows the spectrum of the radial velocity standard II-76 in the globular cluster M13.

${ }^{2}$ IRAF is distributed by the National Optical Astronomy Observatories, which are operated by the Association of Universities for Research in Astronomy, Inc., under contract to the National Science Foundation. 


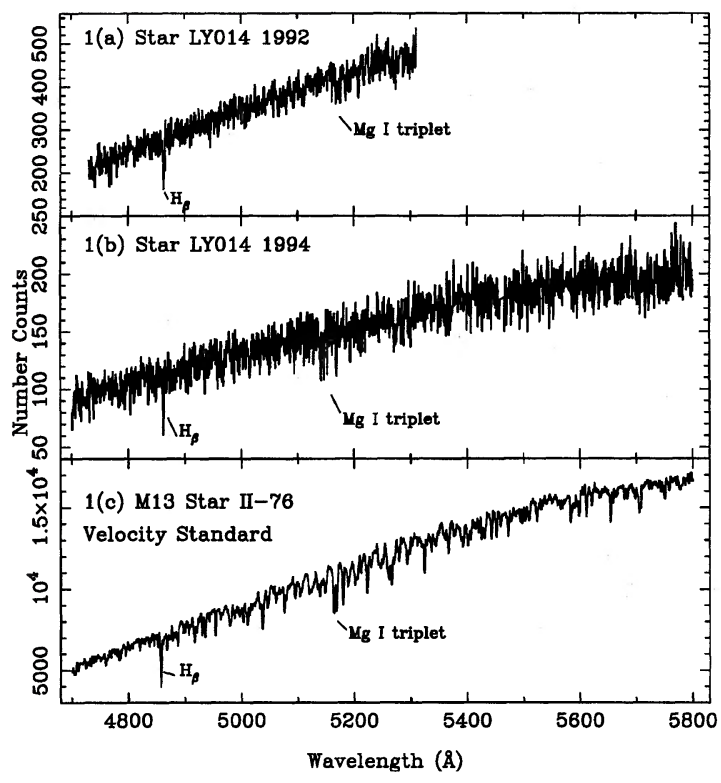

FIG. 1. (a) and (b) show spectra of star LY014 taken in 1992 and 1994. (c) is the spectrum of star II-76 in M13 which serves as a radial velocity standard.

All wavelength calibrations were done only with a set of isolated lines. Typically, we used third-order polynomial fitting and obtained an rms error in the dispersion solution less than $0.04 \AA$, which corresponds to a velocity error of less than $2 \mathrm{~km} \mathrm{~s}^{-1}$. Each stellar spectrum was wavelength calibrated with its own comparison spectra which were taken through the same fiber before and after the star exposure. To make sure spectra taken through different fibers and in different nights have the same wavelength scale, we cross correlated every wavelength calibrated thorium-argon spectrum against all the other Th-Ar spectra. With care in the calibration procedure, we were able to keep the relative shift between all thorium-argon spectra under $2 \mathrm{~km} \mathrm{~s}^{-1}$.

The object spectra were dispersion corrected and binned into log wavelength. Radial velocities for all stars in the sample were obtained with a cross-correlation technique (Tonry \& Davis 1979). Each object spectrum was cross correlated with the template spectra of radial velocity standards. We used the task FXCOR in RV package of IRAF to obtain radial velocity measurements.

\subsection{Radial Velocities}

We have obtained a total of 247 new radial velocities for 77 cluster member giant and subgiant stars in the globular cluster NGC 5053. Of the 77 cluster member stars, 66 stars have multiple radial velocity measurements and are suitable for a binary search. Therefore, we will concentrate only on those 66 stars. Table 2 lists photometry, astrometry and 247 radial velocities for those 77 stars in NGC 5053. The columns record, from left to right, the star's identification names, right ascension, and declination in the epoch of 1950.0, the radial distance from the cluster center in arcseconds, the heliocentric Julian date $(+244000$ in days) of the observation, the heliocentric radial velocity at the date and its corresponding uncertainty in $\mathrm{km} \mathrm{s}^{-1}$, the number of observations, the weighted mean radial velocity, and the external velocity error in a single measurement estimated from the dispersion about the mean, the chi-square for the observations and the probability of obtaining a chi-square at least this large purely due to measurement errors. The final two columns give the star's magnitude and $(B-V)$ color. Some of the photometry listed in Table 2 are from Sandage et al. (Sandage et al. 1977) and the rest are our measurements. To distinguish the stars originally identified in the SKJ paper from the new stars we selected, we combined the same names SKJ used with the prefix S. Table 2 is published in the CD-ROM supplement to this journal due to its large size. Anybody who is interested in Table 2 could also contact LY directly.

In Table 2, the second and any subsequent lines under each object report the radial velocities and the internal errors at the corresponding heliocentric Julian dates. For a combined set of velocities, we calculated $\chi^{2}$ and the weighted mean velocity (for details, see Duquennoy \& Mayor 1991). The $\chi^{2}$ value represents the radial velocity variability over the timespan of three years. To evaluate the significance of the variations represented by $\chi^{2}$, we calculated the probability of having a $\chi^{2}$ at least this large purely due to chance fluctuations with a Gamma function $Q\left(0.5 \nu, 0.5 \chi^{2}\right)$, here $\nu$ is the number of the degree of freedom. Reasonable limits of $\chi^{2}$ probability for identifying significant variations are $0.01-$ 0.001 .

The velocities tabulated in Table 2 are the radial velocities of stars in NGC 5053 relative to star II-76 in M13. The globular clusters M13 and NGC 5053 have cluster systematic velocities of about -246.4 and $43 \mathrm{~km} \mathrm{~s}^{-1}$, respectively (Pryor \& Meylan 1993). Figure 2 shows the histogram of all relative velocities tabulated in Table 2 including non-cluster member velocities. With only cluster members and excluding the variable stars, the mean relative velocity is 291.0 $\mathrm{km} \mathrm{s}^{-1}$ and the standard deviation is $3.7 \mathrm{~km} \mathrm{~s}^{-1}$.

\subsection{Error Analyses}

As shown in Table 1, we usually took multiple exposures of the same set of stars in a single observing run. We measured velocities using individual spectra as well as using the sum of these individual spectra of the same star. For some faint stars, summation of all exposures within a single observing run is required in order to have enough signal to measure velocities.

One problem with summation is that it works fine for almost all types of potential binaries in our sample except binaries with periods of a few days. The shortest period for a binary allowed by the sizes of stars in our sample is $\sim 3$ days. In the case of an edge-on circular orbit with $M_{1}=0.8 \mathscr{U}_{\odot}$, $M_{2}=0.5 \mathscr{C l}_{\odot}$, the expected peak-to-peak radial velocity variation is $\sim 113 \mathrm{~km} \mathrm{~s}^{-1}$. Obviously the binaries with such an orbital configuration can be easily identified by inspecting the velocities measured during the same observing run. We found only one binary candidate ST with radial velocity (individual measurements) variations larger than $30 \mathrm{~km} \mathrm{~s}^{-1}$ over two days. This star is a strong candidate for a shortperiod spectroscopic binary (see Sec. 3.1 for detailed discus- 
TABLE 2. Relative radial velocities of stars in N5053.

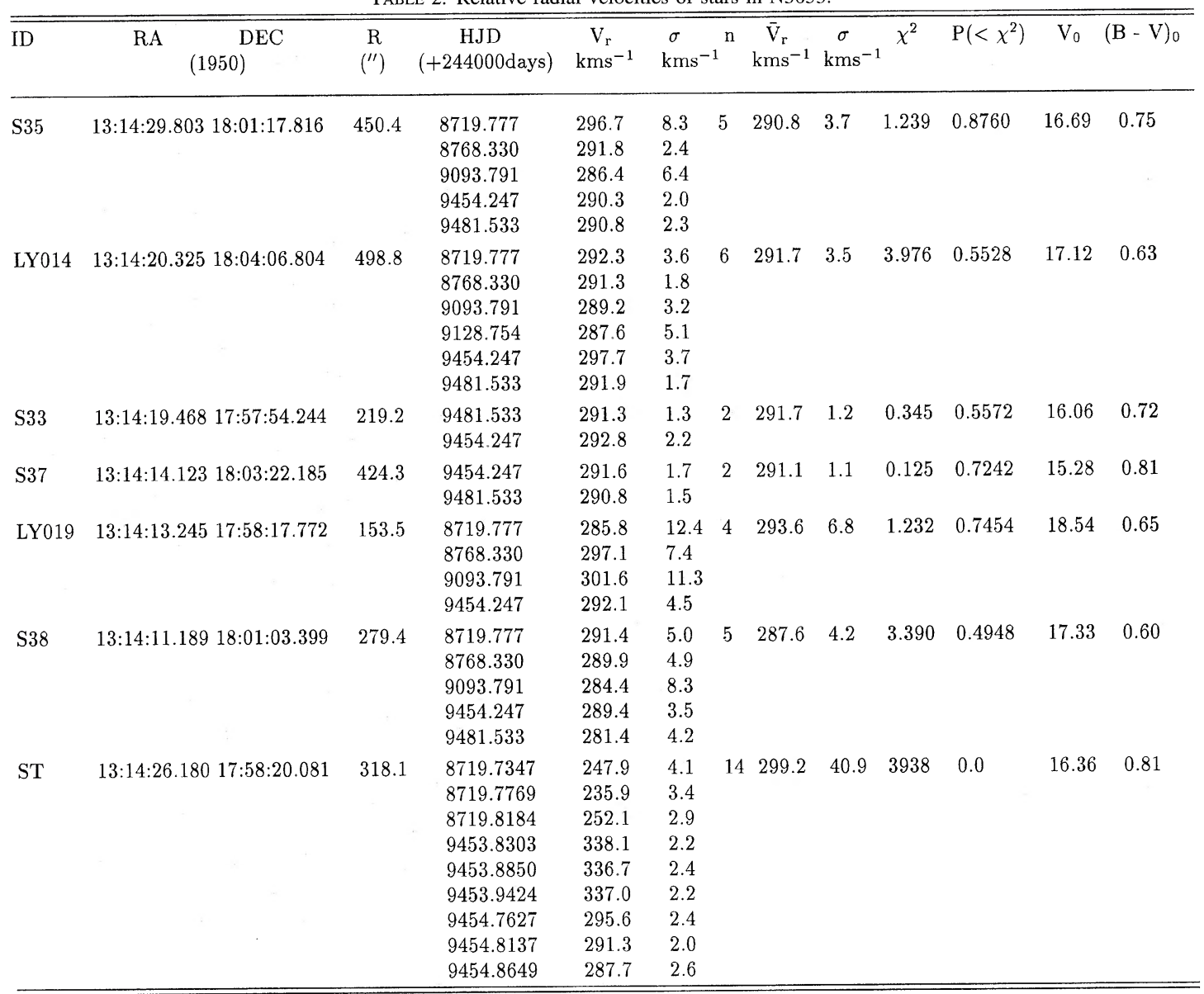

*Table 2 can be found in the AAS CD-ROM Series, Vol. 7, 1996. Only the first page is shown here for form and content.

sion of this system). However, the binaries with periods of a few days and near face-on orbits will not show large radial velocity variations during a single observing run. Summation of spectra will smear out velocity variations of this type of binaries and we can not distinguish them from single stars. The problem is partly inherent for this type of binaries, and partly due to the noisy spectra of the faint stars in our survey. Fortunately, the probability of having this particular type of binaries in our sample is very small $(\leqslant \sim 2 \%)$. The final result of the survey should not be significantly affected by this limitation.

Making a realistic estimate of the internal error for each radial velocity measurement is crucial for identifying the radial velocity variables. The internal velocity errors are evaluated as follows. The quality of a cross correlation can be characterized by a quantity $R$ (for the definition of $R$, see Tonry \& Davis 1979). The larger $R$ is, the more accurate the velocity is. The value of $R$ reflects the signal-to-noise ratio of the object spectrum as well as how well the object spectrum matches with the template spectrum. With this definition of $R$, the internal error is calculated as $\sigma=\sigma_{0} /(1+R)$, here $\sigma_{0}$ is a constant which can be estimate with our data.

The method we used to estimate $\sigma_{0}$ is similar to the one described in Pryor et al. (1988) and Vogt et al. (1995). We took the velocity difference $\Delta v$ between each pair of veloci- ties for those stars with multiple measurements. Then

$$
\sigma_{0}=\frac{\Delta v}{\left[\left(1+R_{1}\right)^{-2}+\left(1+R_{2}\right)^{-2}\right]^{1 / 2}},
$$

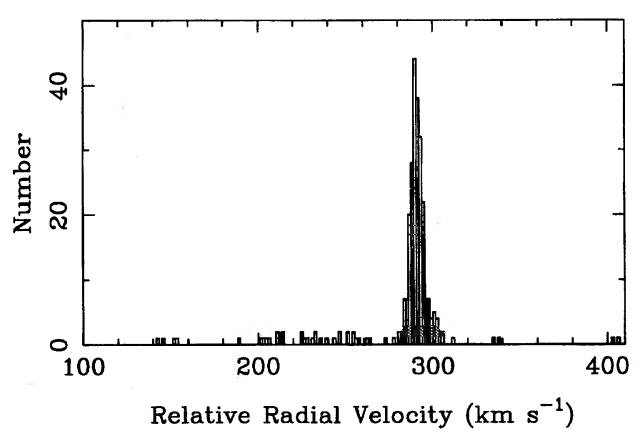

FIG. 2. The histogram of all relative radial velocities measured in our survey including nonmembers of the cluster stars. Each bin is $1.55 \mathrm{~km} \mathrm{~s}^{-1}$ wide. The majority of the stellar radial velocities distributed around the cluster relative velocity. Nonmembers of the cluster stars are clearly distinguished from the member stars by their radial velocities. Also notice that the dispersion around the mean radial velocity is only $3.7 \mathrm{~km} \mathrm{~s}^{-1}$ excluding the measurements from nonmember stars and binary candidates. 


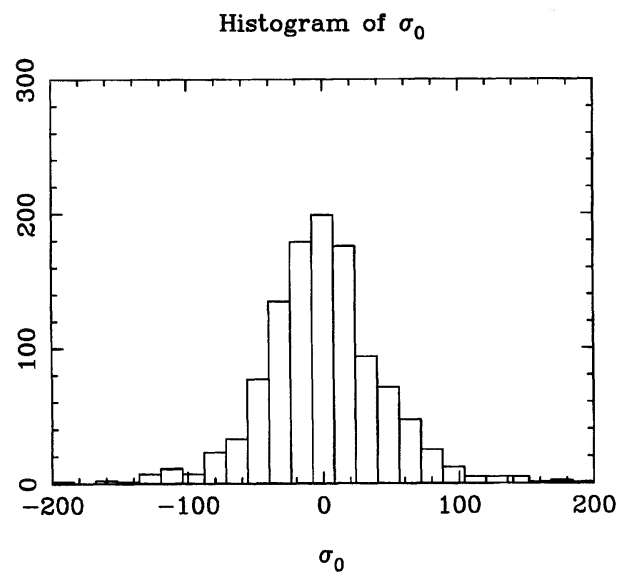

FIG. 3. The distribution of $\sigma_{0}$ derived from our data. See the text for the detailed discussion.

here $R_{1}$ and $R_{2}$ are the Tonry \& Davis values for velocity $v_{1}$ and $v_{2}$, respectively. If velocity errors have a Gaussian distribution, $\sigma_{0}$ is equal to the standard deviation of a normal distribution produced by the above equation. The second way is to compute the velocity difference $\Delta v$ between the weighted mean velocity and a given measurement $v_{i}$. Then $\sigma_{0}$ is

$$
\frac{\left(v_{i}-\vec{v}\right)}{\left[\left(1+R_{i}\right)^{-2}+\left(\sum\left(1+R_{i}\right)^{-2}\right]^{1 / 2}\right.} .
$$

Figure 3 shows the $\sigma_{0}$ distribution. It is roughly gaussian and the standard deviation $\sigma_{0}$ is $44.7 \mathrm{~km} \mathrm{~s}^{-1}$ for the 1992 April observation. For all observed objects, Fig. 4 is the plot of the internal velocity error versus $R$ of each velocity measurement. A separate $\sigma_{0}$ was estimated for each observing run. With the derived $\sigma_{0}$, we got a total $\chi^{2}$ of 185.1 for 196 degrees of freedom for all velocities in our sample excluding non-members and potential variables. The probability of a $\chi^{2}$ larger than this is 0.68 , which is acceptable.

For all stars in our sample, velocity errors listed in Table 2 are calculated using $\sigma_{0}$ estimated with the above method and $R$ values from the summed spectra. A typical error is $\sim 3$ $\mathrm{km} \mathrm{s}^{-1}$, but the worst is as large as $10 \mathrm{~km} \mathrm{~s}^{-1}$. This large variation is partly due to the intrinsic luminosity differences between stars in our sample, and partly because some stars were observed more frequently than others. In addition, the accuracy of fiber positioning is different from star to star and from one observing run to another. With nonuniform velocity measurement errors, it is dangerous to use only the velocity variations between different epochs to represent the true velocity variabilities due to orbital motion of binary stars. The more effective way of selecting binary candidates is to calculate $\chi^{2}$ of all velocity measurements and the probability of obtaining a $\chi^{2}$ value larger than observed by chance.

The velocity zero-point shifts between different epochs were examined by cross-correlating spectra of bright stars in M13. The twilight spectra taken in several epochs were also used to estimate the velocity zero-point shifts. We did

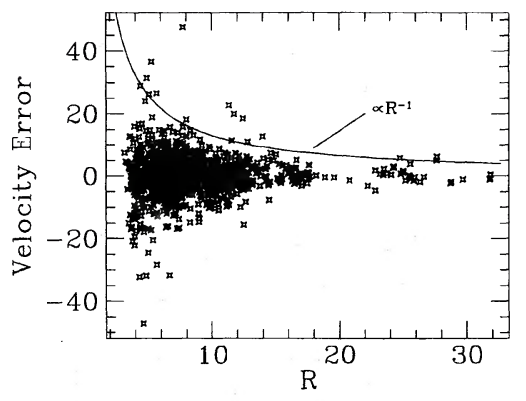

FIG. 4. The plot shows the velocity error vs the Tonry and Davis value $R$. The envelope of the velocity errors in the figure is roughly proportional to $\propto R^{-1}$, as described in Tonry \& Davis (1979).

not find any significant velocity zero-point shifts between different epochs.

\section{RESULTS}

\subsection{Spectroscopic Binary Candidates}

Using the criterion that a binary candidate must have a velocity $\chi^{2}$ such that the probability of obtaining a $\chi^{2}$ larger than this value by chance is less than 0.01 , we identified 6 spectroscopic binary candidates among a sample of 66 cluster member stars. The $\chi^{2}$ probabilities of these six binary candidates indicate that their radial velocity variations are significant over the timespan of $3 \mathrm{yr}$. Of these 6 binary candidates, star S5 was previously found as a binary candidate in the Pryor et al. survey (1991) and star ST is a binary candidate with a very short period and a large-amplitude velocity variation. Table 3 lists these six spectroscopic binary candidates. Figure 5 is a cluster color-magnitude diagram, in which the solid triangles represent five of the six binary candidates discovered by our survey, candidate ST is marked separately with a solid square and the hollow circles show the three brighter binary candidates discovered in the Pryor et al. (1991) survey. Figures 6(a)-6(e) are the detailed finding charts of the six binary candidates. The star is $318.1^{\prime \prime}$ away from the cluster center and located in an isolated environment, as shown in Fig. 6(e). $(B-V)$ color and $V$ magnitude of binary candidate ST are are fairly consistent with those of the cluster member stars.

We fit the velocity data and obtain the orbital solutions for star ST. However, due to the small number of velocity measurements, the orbital solution for star ST is not unique. Figure 7 shows the three possible solutions. In the figure, $P$ is the period, $V_{0}$ the center-of-mass velocity, $K$ the amplitude of the orbital velocity, and $e$ the eccentricity. These possible orbital solutions and its photometric color suggest that star ST is a cluster member. However, the spectrum of this star has a much stronger $\mathrm{Mg} \lambda 5177$ triplet absorption line compared to that of a low-metallicity giant star. The analyses of the $\mathrm{Mg} \lambda 5177$ line strength verses the photometric color (for the details of the method, see Faber et al. 1985; Gorgas et al. 1993) indicate that the spectrum of star ST is similar to that of a metal-rich dwarf. But considering that star ST is a very short-period spectroscopic binary, we conclude the parcularity of its absorption line strength could result from the pos- 
TABLE 3. Spectroscopic binary candidates in N5053.

\begin{tabular}{|c|c|c|c|c|c|c|c|c|c|c|}
\hline ID & $\begin{array}{c}\text { HJD } \\
(+244000 \text { days })\end{array}$ & $\begin{array}{c}\mathrm{V}_{\mathrm{r}} \\
\mathrm{kms}^{-1}\end{array}$ & $\begin{array}{l}\sigma \\
\mathrm{kms}^{-1}\end{array}$ & ${ }_{1}^{n}$ & $\begin{array}{c}\overline{\mathrm{V}}_{\mathrm{r}} \\
\mathrm{kms}^{-1}\end{array}$ & $\begin{array}{c}\sigma \\
\mathrm{kms}^{-1}\end{array}$ & $\chi^{2}$ & $\mathrm{P}\left(<\chi^{2}\right)$ & $\mathrm{V}_{0}$ & $(B-V)_{0}$ \\
\hline S54 & $\begin{array}{l}8719.777 \\
8768.330 \\
9093.791 \\
9128.754 \\
9454.247 \\
9481.533\end{array}$ & $\begin{array}{l}277.1 \\
292.2 \\
295.4 \\
281.0 \\
294.0 \\
302.6\end{array}$ & $\begin{array}{l}3.1 \\
2.2 \\
3.2 \\
4.7 \\
3.4 \\
3.4\end{array}$ & 6 & 291.1 & 9.6 & 39.236 & 0.0000 & 16.63 & 0.65 \\
\hline S109 & $\begin{array}{l}8719.777 \\
8768.330 \\
9093.791 \\
9128.754 \\
9454.247 \\
9481.533\end{array}$ & $\begin{array}{l}288.3 \\
291.6 \\
288.6 \\
304.9 \\
300.2 \\
303.2\end{array}$ & $\begin{array}{l}3.7 \\
3.6 \\
4.0 \\
4.9 \\
3.7 \\
2.2\end{array}$ & 6 & 297.5 & 7.6 & 23.30 & 0.0003 & 16.02 & 0.75 \\
\hline SAB & $\begin{array}{l}8719.777 \\
8768.330 \\
9093.791 \\
9128.754 \\
9454.247 \\
9481.533\end{array}$ & $\begin{array}{l}291.5 \\
295.2 \\
306.1 \\
291.2 \\
300.3 \\
289.2\end{array}$ & $\begin{array}{l}2.9 \\
2.7 \\
4.9 \\
9.2 \\
2.4 \\
2.6\end{array}$ & 6 & 295.1 & 6.5 & 16.60 & 0.0053 & 16.76 & 0.66 \\
\hline \$91 & $\begin{array}{l}8719.777 \\
8768.330 \\
9454.247 \\
9481.533\end{array}$ & $\begin{array}{l}291.5 \\
310.9 \\
291.8 \\
298.4\end{array}$ & $\begin{array}{l}4.3 \\
4.9 \\
2.6 \\
2.9\end{array}$ & 4 & 296.1 & 9.4 & 13.63 & 0.0035 & 16.97 & 0.70 \\
\hline S5 & $\begin{array}{l}8719.777 \\
8768.330 \\
9093.791 \\
9454.247 \\
9481.533\end{array}$ & $\begin{array}{l}304.3 \\
294.5 \\
280.3 \\
287.5 \\
300.9\end{array}$ & $\begin{array}{l}2.8 \\
3.1 \\
6.0 \\
2.1 \\
2.4\end{array}$ & 5 & 295.1 & 9.9 & 35.86 & 0.0000 & 15.67 & 0.74 \\
\hline ST & $\begin{array}{l}8719.7347 \\
8719.7769 \\
8719.8184 \\
9453.8303 \\
9453.8850 \\
9453.9424 \\
9454.7627 \\
9454.8137 \\
9454.8649 \\
9480.6831 \\
9480.7310 \\
9480.7857 \\
9482.7090 \\
9482.7564\end{array}$ & $\begin{array}{l}247.9 \\
235.9 \\
252.1 \\
338.1 \\
336.7 \\
337.0 \\
295.6 \\
291.3 \\
287.7 \\
337.6 \\
338.7 \\
335.4 \\
254.2 \\
254.7\end{array}$ & $\begin{array}{l}4.1 \\
3.4 \\
2.9 \\
2.2 \\
2.4 \\
2.2 \\
2.4 \\
2.0 \\
2.6 \\
2.0 \\
2.1 \\
2.3 \\
1.7 \\
1.8\end{array}$ & 14 & 299.2 & 40.9 & 3938 & 0.0 & 16.36 & 0.81 \\
\hline
\end{tabular}

Notes: As in Table 2, see the text for the detailed description of the table.

sible interaction between the two components of the binary system; star ST is probably a cluster member. We should point out that if star ST is a cluster member giant and if its period is around three to five days, this binary system must be on the verge of Roche lobe overflow since the primary star has a fairly large radius.

\subsection{Modeling the Binary Frequency ${ }^{3}$}

In any spectroscopic survey for binary stars, the probability of detecting a binary depends not only on the binary

${ }^{3}$ Here the binary frequency is defined as the ratio of the number of binary candidates to the total number of "stars," here "stars" includes both single stars and binary stars; and a binary is counted as "one star." frequency, but also on a set of unknown binary orbital parameters such as period, eccentricity, mass ratio, orbital phase, and inclination angle of the binary system. Unfavorable binary orbital configurations would cause a fraction of binary systems to be missing from our detection sample. Thus, to properly interpret the measurements in our survey, we use Monte Carlo simulation methods to generate a large number of simulated radial velocity data sets and compare them with the measured radial velocities. We calculate from the synthetic data sets the fraction of binaries missed in our survey, then correct the observed binary frequency. We applied the same criterion used in the survey to the synthetic data for identifying binary candidates. This criterion is that 


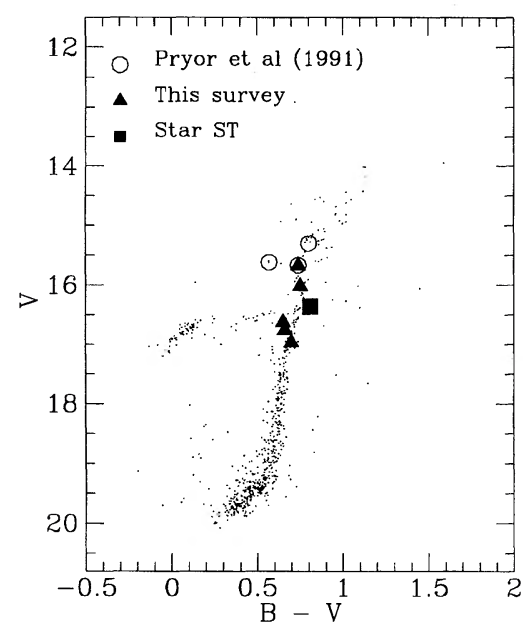

FIG. 5. This shows the cluster color-magnitude diagram, where the solid triangles show five of the six binary candidates discovered in this survey, the solid square indicates the short-period binary candidate ST, and the open circles represent the candidates found previously by Pryor et al. (1991).

the probability of obtaining a $\chi^{2}$ larger than observed by chance has to be less than 0.01 .

An additional statistical method is to use a KolmogorovSmirnov (K-S) test to compare the cumulative distributions of maximum velocity differences for the real data to the mean distribution determined from a large number of simulated data sets. This method has been used in several surveys, such as Harris \& McClure (1983), Pryor et al. (1988), and Côte et al. (1994). We will discuss in detail the applicability of this method to our data in Sec. 4.3.

\subsubsection{The binary models}

The simulation code for generating synthetic radial velocities was generously provided to us by Dr. C. Pryor. Some modifications have been made during our calculations. In solving Kepler's equations, we made the following assumptions: the binary period, mass ratio, and eccentricity distribution functions were taken as the ones in Duquennoy \& Mayor (1991, thereafter DM), which were derived for G-type dwarf stars in the solar neighborhood. The orbital longitude $\omega$ and the initial orbital phase were drawn randomly between 0 and $2 \pi$ from a uniform distribution. $\cos (i)$, where $i$ is the inclination angle of the orbital plan to the line of sight, was chosen randomly from 0 to 1 with a uniform distribution. Of course, the mass of the primary is assumed to be $0.8 \mathscr{L b}_{\odot}$. Our survey is sensitive to binaries with the minimum and maximum periods of 3 days and 10 years respectively, and with mass ratio larger than 0.125 .

The effect of mass transfer was considered in our simulations by eliminating the binary system whenever the two stars get closer than the critical Roche lobe radius. The radius of the primary was calculated from its photometry using the Revised Yale Isochrones (Green et al. 1987). Figure 8 shows the stellar radius versus absolute visual magnitude (reddening corrected) for the stars in our sample. In Fig. 8, the solid line is calculated for the metallicity of -2.2 from the models, the solid triangles represent the stars in our sample with the adopted distance modulus $(m-M)_{V 0}$ of 16.08 mag and $\mathrm{E}(B-V)$ of 0.06 mag (Fahlman et al. 1991).

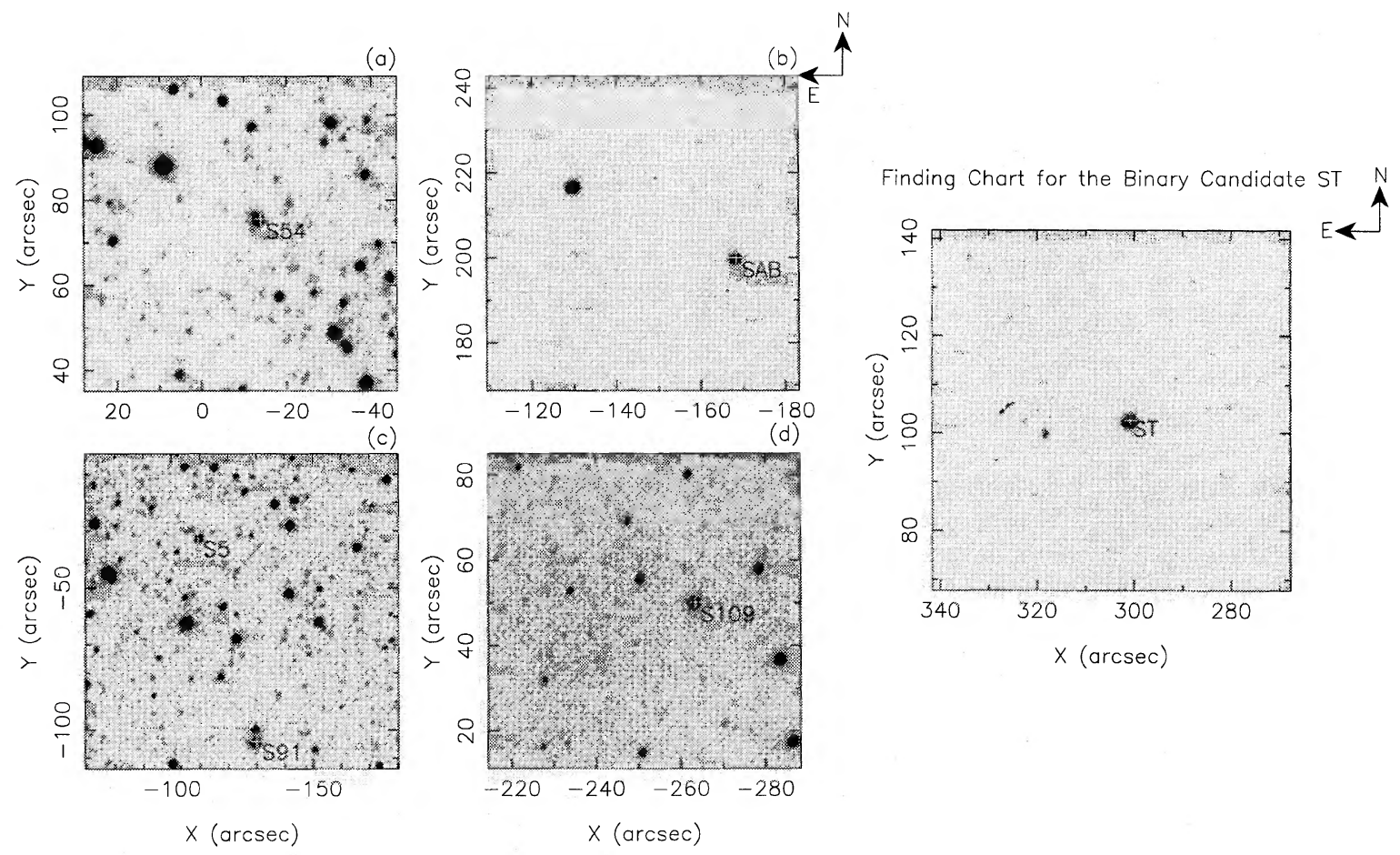

FIG. 6. The detailed finding charts for the six binary candidates. All objects are plotted relative to the position with R.A. (1950)=13:14:5.070 and Dec. $(1950)=17: 56: 38.00$. The axes are in units of arcsecond, the north and the east are indicated in the plot. 


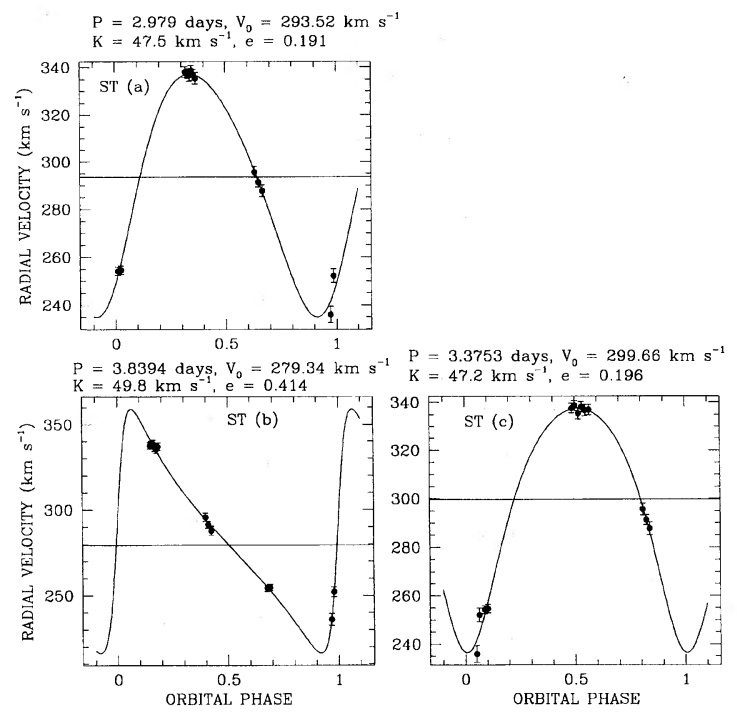

FIG. 7. The plots show the three possible orbital solutions fitted to the available data.

Also shown in the figure with the hollow squares are the empirical radii for giants in the clusters M92 by Cohen et al. (1987) based on broadband infrared photometry and narrowband $\mathrm{CO}$ and $\mathrm{H}_{2} \mathrm{O}$ indices. The globular cluster $\mathrm{M} 92$ has a metallicity $[\mathrm{Fe} / \mathrm{H}]$ of -2.2 , very similar to NGC 5053 .

We did not consider the effect of asymptotic giant branch in our calculations because in our sample less than $24 \%$ of stars are above the Horizontal branch.

\subsubsection{The binary frequency}

To convert the binary discovery fraction of $0.084(5.5 /$ 65.5 , giving candidate ST 0.5 weight due to some ambiguity of its cluster membership) to the true binary frequency $f_{b}$, we estimate the incompleteness correction using Monte

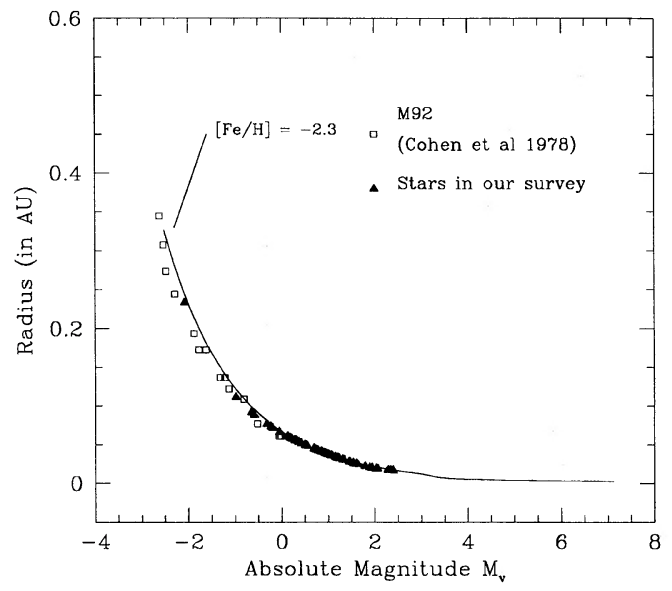

FIG. 8. This is a plot of the stellar radius vs. the luminosity for stars in globular clusters. The curve is generated using the Revised Yale Isochrones and Luminosity Functions (Green et al. 1987) with $Y=0.2$ and an age of 15 Gyr. The solid triangles represent the stars in our sample, and open squares are the measurements for stars in M92 by Cohen et al. (1987).

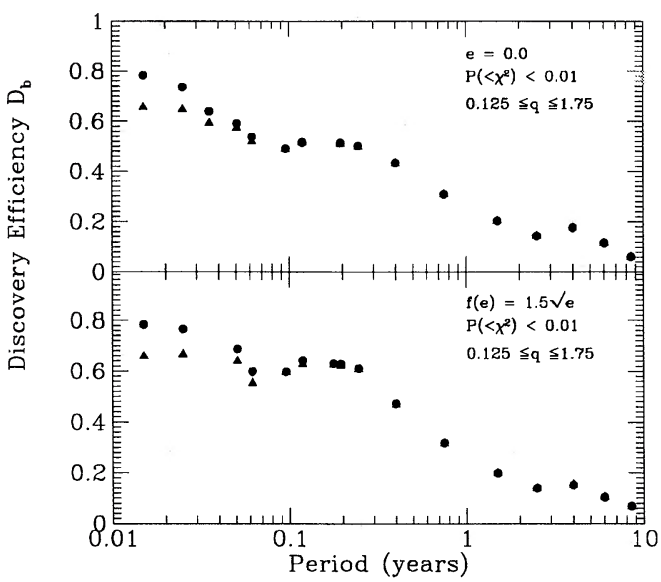

FIG. 9. (a) and (b) show the binary discovery efficiency $D_{b}$ as a function of period $P$ (in days) for two different binary models. In both figures the solid triangles and the solid dots represent the simulations with and without considering the mass transfer effect, respectively. Note that each data point in the plots is the mean of 1000 simulation tries.

Carlo simulations. This correction is also called the binary discovery efficiency $D_{b}$, which is defined as the fraction of the "discovered" binaries in the synthetic velocity data if we assume all 66 stars as binary systems. Radial velocities of each binary were calculated using the binary models described in Sec. 3.2.1 at the actual observation dates. Velocity noises were drawn randomly from Gaussian distributions with the mean of zero and standard deviations of the real velocity measurement errors. By applying the same binary identifying criterion used in the survey, we obtained the fraction of "discovered" binaries in the simulated data. Figures 9 (a) -9 (b) show the binary discovery efficiency $D_{b}$ as a function of period $P$ in days for the binary models with circular orbits and with eccentricity distribution $f(e)=1.5 \sqrt{ } e$, respectively. Notice that each data point in the plots is the mean of 1000 simulation tries. Here we assume the primary star in a binary system to have $0.8 \mathscr{C}_{\odot}$, and a mass ratio $q$ (defined as $\left.M_{2} / M_{1}\right)$ in the range of $0.125-1.75$. This implies that the secondary could be anything from a $0.1 \mathscr{M}_{\odot}$ low-mass star to a $1.4 \mathscr{L}_{\odot}$ heavy neutron star. In both plots, the solid triangles and dots represent simulations with and without the effect of mass transfer, respectively. It is clearly shown in the figures that the biasing of the discovered binary orbits by the size of the giants is significant at the short period end. This bias is higher for stars with brighter magnitudes, which is the major reason that the previous surveys could not detect any binary with period shorter than 40 days.

In the case of nonzero eccentricity orbits, the average binary discovery efficiency is about $29 \%$ for binaries with 3 days $\leqslant P \leqslant 10$ years and $0.125 \leqslant q \leqslant 1.75$. For binaries with the same period and mass ratio range, the inferred true binary frequency $f_{b}$ is $0.084 / D_{b}$, i.e., $29 \%$. In the case of circular orbits, the corresponding binary frequency for systems with periods and mass ratios in the same ranges is $26 \%$. The discovery efficiency varies as the limits of binary period and mass ratio change. A binary with a very low mass companion is usually difficult to detect even if the period is short. 
For instance, the primary star in a binary with a period of 100 days shows a maximum radial velocity change of 10 $\mathrm{km} \mathrm{s}^{-1}$ in the case of an edge-on circular orbit and $M_{1}=0.8$ $\mathscr{C}_{\odot}, M_{2}=0.1 \mathscr{M}_{\odot}$. If we choose the sensitivity limits of our survey as 3 days $\leqslant P \leqslant 10$ years and $0.3 \leqslant q \leqslant 1.75$, the binary discovery efficiency is $35 \%$ and $37 \%$ for the binary models with noncircular and circular orbits, respectively. The corresponding binary frequency $f_{b}$ in the cluster is $24 \%$ and $23 \%$, respectively.

\section{DISCUSSION}

\subsection{An Alternative Period Distribution Function in Globular Clusters}

The binary frequency estimated above depends on the adopted period distribution in the simulations. An alternative period distribution has a constant number of binaries per unit logarithmic period interval. This so-called "flat" period distribution is a crude representation of the survey by Abt \& Levy (1976). The "flat" distribution produces more shortperiod binaries and less long period ones for $3 \mathrm{~d} \leqslant P \leqslant 10 \mathrm{yr}$ than the DM period distribution. Obviously, it also gives a somewhat higher binary discovery efficiency for our survey. Specifically, with an average discovery efficiency of $36 \%$ derived from the "flat" distribution, we obtained a binary frequency of $23 \%$ for systems with $3 \mathrm{~d} \leqslant P \leqslant 10 \mathrm{yr}$, $0.125 \leqslant q \leqslant 1.75$, and $f(e)=1.5 \sqrt{ } e$. Similarly in the case of circular orbits, the inferred binary frequency is $21 \%$. For the mass ratio range of $0.3 \leqslant q \leqslant 1.75$, the derived binary frequencies are $19 \%$ and $22 \%$ for circular and eccentric orbits, respectively. The binary frequency derived by using the "flat" period distribution is smaller than using the DM period distribution in the simulations. The binary period distribution in globular clusters perhaps bears more similarities with ones in open clusters and among Pop. II low-metallicity halo stars. Many extensive programs of studying binary stars in open clusters and among low-metallicity halo stars (Carney \& Latham 1987; Latham et al. 1988, 1992) should shed light on the properties of binary populations in globular clusters.

\subsection{The Effect of Stellar Encounters}

We did not take into account the effect of stellar encounters in our calculations. This effect can alter the shape of the primordial distributions of binary orbital elements in globular clusters. As briefly discussed in Sec. 2.1, this effect is not significant in very low density clusters such as NGC 5053 since the cluster half-mass relaxation time scale is comparable to a Hubble time scale $T_{h}$. However, of the eight globular clusters in which radial velocity surveys for binary stars have been carried out by various groups (Hut et al. 1992), six have cluster central relaxation time scales $T_{r h}$ much shorter than $T_{h}$ of $14 \mathrm{Gyr}$. These six clusters include M3, M13, 47 Tuc, M2, M71, and NGC 3201, where $T_{r h}$ is in the range of $10^{8}-10^{9} \mathrm{yr}$. NGC 3201 and M71 have particularly short $T_{r h}$ of only 100 million years in spite of their apparent low central densities.

It is a rather complicated problem to characterize quantitatively the change in the primordial period distribution due to dynamical evolution. A tremendous amount of theoretical

\begin{tabular}{l} 
TABLE 4. Kolmogorov-Smirnov probabilities for model acceptance. \\
\hline
\end{tabular}

computations have been devoted to this subject (Phinney \& Sigurdsson 1991; McMillan \& Hut 1994; Sigurdsson \& Phinney 1995). The detailed $N$-body simulations (McMillan et al. 1991) show that binary-binary encounters are a very effective binary-destruction process. Most binary destruction occurs within a few core radii of the cluster center. In addition, some binaries can be ejected to the outer parts of a cluster due to large recoil during binary-binary encounters in the core. Thus, in dynamically evolved clusters such as M71 it is perilous to assume the field period distribution.

Although it is premature to draw any comprehensive conclusions about the binary frequency dependence on the dynamical properties of globular clusters, it is illuminating to compare the results from various surveys for binary stars in different globular clusters. As first pointed out by Pryor et al. (1991; Hut et al. 1992), the binary discovery rate in NGC 5053 is notably higher than other clusters where binary searches have been carried out. The result of our survey appears to further support Pryor's conclusion. This is perhaps associated with the fact that NGC 5053 is a dynamically young cluster.

\subsection{Kolmogorov-Smirnov $(K-S)$ Tests}

As Harris \& McClure (1983) first pointed out, small number statistics are important in estimating binary frequencies in globular clusters. Subsequently a sophisticated and quantitative statistical method-the Kolmogorov-Smirnov testhas been employed in many surveys. $\mathrm{K}-\mathrm{S}$ tests were applied to the cumulative distributions of maximum velocity difference obtained from both simulated and real data. A K-S statistic is useful for rejecting the null hypothesis that two data sets are drawn from the same parent distribution.

We applied $\mathrm{K}-\mathrm{S}$ statistics to our measured velocities and the simulated data. In Table 4, we tabulated the confidence level at which the null hypothesis of the simulated data and real data drawn from the same distribution is accepted. $\mathrm{K}-\mathrm{S}$ tests show that the binary frequency in NGC 5053 is close to $25 \%$, and the hypothesis that the binary frequency is higher than $50 \%$ is rejected with confidence higher than $85 \%$. However, it is noticed in Table 4 that with our data $\mathrm{K}-\mathrm{S}$ tests are not very effective in rejecting the cases with different binary frequencies. This problem results from some of the large errors in our velocity measurements. As described previously, the synthetic data were generated using the actual ve- 
locity errors. The corresponding cumulative distribution of maximum velocity variation is primarily controlled by a few large errors. $\mathrm{K}-\mathrm{S}$ tests have been applied more effectively by Pryor et al. (1988) and Côte et al. (1994) to their data with the radial velocity errors are as small as $1 \mathrm{~km} \mathrm{~s}^{-1}$. They have derived a binary frequency in the range of $10 \%-20 \%$ for binaries with $0.1 \mathrm{yr} \leqslant P \leqslant 20 \mathrm{yr}$.

We would like to thank The Kenneth T. and Eileen L. Norris Foundation and especially Kenneth Norris for their generous support in building the Norris spectrograph.
Michael Doyle, John Henning, and Juan Carrasco of the Palomar Observatory staff are thanked for providing excellent service during the observations. We wish to thank Tad Pryor for his unreserved help and interesting discussions about astronomy on many occasions. The Norris Spectrograph was built by Bev Oke, Judith Cohen, and Donald Hamilton; we thank Donald Hamilton and Todd Small for their help. We also wish to thank an anonymous referee for providing many excellent suggestions which have helped to improve the paper.

\section{REFERENCES}

Abt, H. A., \& Levy, S. G. 1976, ApJS, 30, 273

Carney, B. W., \& Latham, D. W. 1987, AJ, 93, 116

Cohen, J. G., Frogel, J. A., \& Persson, S. E. 1978, ApJ, 222, 165

Côte, P., Welch, D. L., Fischer, P., Da Costa, G. S., Tamblyn, P., Seitzer, P., \& Irwin, M. J. 1994, ApJS, 90, 83

Djorgovski, S., \& King, I. R. 1986, ApJ, 305, L61

Djorgovski, S. 1992, in Structure and Dynamics of Globular Clusters, ASP

Conf. Ser. 50, edited by S. G. Djorgovski and G. Meylan (ASP, San Francisco), p. 357

Duquennoy, A., \& Mayor, M. 1991, A\&A, 248, 485

Faber, S. M., Friel, E. D., Burstein, D., \& Gaskell, C. M. 1985, ApJS, 57, 711

Fahlman, G. G., Richer, H. B., \& Nemec, J. 1991, ApJ, 380, 124

Gao, B., Goodman, J., Cohn, H., \& Murphy, B. 1991, ApJ, 370, 567

Gorgas, J., Faber, S. M., Burstein, D., Gonzalez, J. J., Courteau, S., \& Prosser, C. 1993, ApJS, 86, 153

Green, E. M., Demarque, P., \& King, C. R. 1987, The Revised Yale Isochrones and Luminosity Functions (Yale University Observatory, New Haven)

Grindlay, J. E., Hertz, P., Steiner, J. E., Murray, S. S., \& Lightman, A. P. 1984, ApJ, 282, L13

Gunn, J. E., \& Griffin, R. F. 1979, AJ, 84, 752

Kaluzny, J., \& Krzeminski, W. 1993, MNRAS, 264, 785

Kulkarni, S. R., Anderson, S. B., Prince, T. A., \& Wolszczan, A. 1991, Nature, 349, 47

Hamilton, D., Oke, J. B., Carr, M. A., Cromer, J., Harris, F. H., Cohen, J G., Emery, E., \& Blakee, L. 1993, PASP, 105, 1308

Harris, H. C., \& McClure, R. D. 1983, ApJ, 265, L77

Heggie, D. C., \& Aarseth, S. J. 1992, MNRAS, 257, 513

Hut, P., McMillan, S. L. W., Goodman, J., Mateo, M., Phinney, E. S., Richer, H. B., Verbunt, F., \& Weinberg, M., 1992, PASP, 104, 981
Latham, D. W., et al. 1992, AJ, 104, 774

Latham, D. W., Mazeh, T., Carney, B. W., McCrosky, R. E., Stefanik, R. P., \& Davis, R. J. 1988, AJ, 96, 567

Lupton, R. H., Gunn, J. E., \& Griffin, R. F. 1987, AJ, 93, 1114

Mateo, M., Harris, H. C., Nemec, J., \& Olszewski, E. W. 1990, AJ, 100, 469.

McKenna, J., \& Lyne, A. G. 1988, Nature, 336, 226

McMillan, S. L. W., \& Hut, P. 1994, ApJ, 427, 793

McMillan, S. L. W., Hut, P., \& Makino, J. 1991, ApJ, 372, 111

Phinney, E. S. 1992, Philos. Trans. R. Soc. London A, 341, 39

Phinney, E. S., \& Sigurdsson, S. 1991, Nature, 349, 220

Phinney, E. S. 1996, in The Origins, Evolution, and Destinies of Binary Stars in Clusters, ASP Conf. Ser. 90, edited by Eugene F. Milone (ASP, San Francisco) (in press)

Pryor, C. P., Latham, D. W., \& Hazen, M. L. 1988, AJ, 96, 123

Pryor, C. P., \& Meylan, G. 1993, in Structure and Dynamics of Globular Clusters, ASP Conf. Ser. 50, edited by S. G. Djorgovski and G. Meylan (ASP, San Francisco), p. 357

Pryor, C., Schommer, R. A., \& Olszewski, E. W. 1991, in The Formation and Evolution of Star Clusters, ASP Conf. Ser. 13, edited by K. Janes (ASP, San Francisco), p. 121

Sandage, A. R., Katem, B., \& Johnson, H. L. 1977, AJ, 82, 389

Sigurdsson, S., \& Phinney, E. S. 1995, ApJS, 99, 609

Tonry, J., \& Davis, M. 1979, AJ, 84, 1511

Vesperini, E., \& Chernoff, D. F. 1994, ApJ, 431, 231

Vogt, S., Mateo, M., Olszewski, E. W., \& Keane, M. J. 1995, AJ, 109, 151

Yan, L., \& Mateo, M. 1994, AJ, 108, 1810

Yan, L., \& Reid, N. I. 1995, MNRAS, 279, 751 